\title{
BAUMGART, Peter, KROENER, Bernhard R., STÜBIG, Heinz, Die preußische Armee zwischen Ancien Régime und Reichsgründung
}

Bertrand Goujon

\section{(2) OpenEdition \\ Journals}

Édition électronique

URL : http://journals.openedition.org/ifha/1747

DOI : 10.4000/ifha. 1747

ISSN : 2198-8943

Éditeur

IFRA - Institut franco-allemand (sciences historiques et sociales)

Référence électronique

Bertrand Goujon, «BAUMGART, Peter, KROENER, Bernhard R., STÜBIG, Heinz, Die preußische Armee zwischen Ancien Régime und Reichsgründung », Revue de l'IFHA [En ligne], Date de recension, mis en ligne le 01 janvier 2009, consulté le 22 septembre 2020. URL : http://journals.openedition.org/ifha/ 1747 ; DOI : https://doi.org/10.4000/ifha. 1747

Ce document a été généré automatiquement le 22 septembre 2020

(CIFHA 


\title{
BAUMGART, Peter, KROENER, Bernhard R., STÜBIG, Heinz, Die preußische Armee zwischen Ancien Régime und Reichsgründung
}

\author{
Bertrand Goujon
}

Réunissant diverses contributions aux $29 \mathrm{e}$ et $31 \mathrm{e}$ journées annuelles de l'Arbeitsgemeinschaft zur preußischen Geschichte, cet ouvrage collectif revient sur cet élément central qu'a été l'armée dans la construction et l'affirmation de l'État prussien. Tout en s'inscrivant dans la lignée du renouvellement historiographique que connaît l'histoire militaire depuis une vingtaine d'années, il complète par ailleurs le débat concernant la spécificité et la place de la Prusse en Allemagne en le replaçant opportunément dans le long terme. Les divers articles offrent en effet un vaste panorama de l'armée prussienne, du règne de Frédéric-Guillaume Ier (1713-1740) à la proclamation du Reich allemand et à la loi militaire (Reichsmilitärgesetz) de 1874. Loin de faire prévaloir les phases glorieuses - jusqu'alors volontiers privilégiées dans les travaux des historiens et ici délibérément passées sous silence en tant que telles - que furent l'époque frédéricienne ou les guerres de Libération, ces contributions infléchissent sensiblement certains raccourcis et poncifs en mettant l'accent sur des jalons jusqu'à présent méconnus ou négligés. Sans réaliser un balisage continu et exhaustif de la période étudiée, elles soulignent des phénomènes complexes de transition au sein d'une institution dont le recrutement, le mode de fonctionnement, les pratiques, les représentations ainsi le rapport au pouvoir politique et à la société dans son ensemble sont moins immuables que ne l'a longtemps fait présupposer une conception anhistorique $\mathrm{du}$ militarisme comme intrinsèquement constitutif de l'identité prussienne.

L'ordre de présentation des contributions se veut globalement chronologique. On peut à ce titre regretter la césure opérée de manière radicale entre les XVIIIe et XIXe s., qui font l'objet des deux grandes parties de l'ouvrage : elle apparaît d'ailleurs d'autant 
moins pertinente que plusieurs articles s'inscrivent précisément à la charnière des deux siècles. Cette rigidité dans la présentation formelle de l'ouvrage contraste singulièrement avec son contenu, qui se caractérise par un foisonnement des approches. Les apports de la biographie, de la prosopographie, de la sociologie historique, de l'histoire du droit ou du linguistic turn s'y révèlent dans leur complémentarité et contribuent à renouveler la connaissance d'un des objets les plus " traditionnels » de l'histoire.

En mettant l'accent sur les années de jeunesse du futur Frédéric-Guillaume Ier, durant lesquelles le prince héritier de la couronne de Prusse prend le contre-pied du modèle absolutiste baroque incarné par son père Frédéric Ier tout en étant pleinement intégré dans les milieux du pouvoir dès avant son avènement, P. BAUMGART remet ainsi en question le mythe du " roi sergent " - une image simplificatrice d'un souverain dont l'activité réformatrice s'étend également aux questions financières et administratives, visant à faire de la Prusse une grande puissance à l'échelle germanique tout en s'inscrivant dans une durable fidélité à l'empereur Charles VI. C'est également pour une démarche biographique qu'opte M. ROHRSCHNEIDER avec la figure de Léopold Ier d'Anhalt-Dessau, artisan de la modernisation de l'armée prussienne dans le premier tiers du XVIIIe s. Ses attaches généalogiques, sa formation et les étapes de sa carrière militaire sont autant de clés pour comprendre les modalités selon lesquelles il s'inspire du modèle des réformes militaires menées aux Pays-Bas par la Maison d'Orange (en matière d'art de la guerre et de philosophie politique), même si son rôle de médiateur dans ce transfert militaro-culturel ne se réduit pas à une simple imitation et n'exclut nullement l'intégration d'influences françaises ou suédoises. Quant à H.S., l'article qu'il consacre à trois auteurs issus des rangs de l'armée prussienne (Georg Heinrich von Berenhorst, Heinrich Dietrich von Bülow et Gerhard von Scharnhorst) mêle des éléments de type biographique à une étude comparée des argumentaires, très critiques à l'égard d'une institution militaire post-frédéricienne qui apparaît obsolète aux observateurs aguerris. Si Berenhorst réhabilite la notion de hasard en matière de tactique militaire et insiste sur la nécessité d'une formation plus concrète du corps des officiers, Bülow propose au contraire une véritable mathématisation de l'art militaire et une mobilisation de l'ensemble de la population en vue de la guerre - un thème particulièrement cher à Scharnhorst qui, fort de son expérience des guerres contre la France révolutionnaire, défend le concept d'une armée nationale, secondée d'une milice et combattant pour l'indépendance de la monarchie prussienne avec un enthousiasme comparable à celui des soldats français mobilisés par le mot d'ordre de la patrie en danger comme le rappelle M. SIKORA.

À l'instar de Scharnhorst, loin de s'enfermer dans une approche trop exclusivement prusso-centrée, certains auteurs ont judicieusement adopté une perspective comparée et transnationale riche d'enseignements. Menée B.R. KROENER, l'étude du recrutement social et du mode de promotion au sein des armées prussiennes, françaises et autrichiennes au fil du XVIIIe s. montre ainsi clairement qu'il n'existe pas véritablement de spécificité prussienne en la matière, tant reste partout prégnante la domination d'une partie de la noblesse au sein d'institutions militaires qui sont partie prenante de leur conscience de groupe. Quant à C. ALLMAYER-BECK, il souligne combien, après la guerre de Sept Ans, les représentations que suscite l'armée prussienne dans les rangs des officiers autrichiens (du capitaine de hussards Jocob de Cognazo au Feldmarschall comte Lacy, au duc Albert de Saxe-Teschen ou à l'archiduc Charles) sont marquées du sceau de la critique, méfiante tant qu'il s'agit de tirer les 
enseignements nécessaires à la réforme des armées impériales, puis franchement méprisante lorsque, dans le cadre de l'alliance contre-révolutionnaire de 1792-1795, apparaissent au grand jour les divergences stratégiques ainsi que les défaillances en matière de recrutement et de logistique. Bien différente est l'image que se font leurs successeurs dans les armées impériales après la défaite de Sadowa, comme le signale J. ANGELOW : à l'arrogance cisleithanienne de la première moitié du XIXe s. succède en 1866 un revanchisme qui suppose une capacité à tirer les leçons de la supériorité militaire prussienne, mais qui est grevé par le caractère multinational de la monarchie habsbourgeoise et les débats politiques concernant l'avenir de l'espace germanique jusqu'à la proclamation de l'Empire allemand.

La question de la militarisation de l'État prussien fait également l'objet d'une attention particulière. R. STRAUBEL relativise ainsi la place qu'occupent les militaires sortis du rang dans la fonction publique durant la seconde moitié du XVIIIe s. : si certains emplois (notamment subalternes dans l'administration fiscale ou douanière) attirent nombre d'anciens officiers, les postes les plus prestigieux comme ceux de Finanzrat et même de Kriegsrat reviennent presque exclusivement à des civils dotés d'une solide formation juridique et intellectuelle. Un début de carrière dans les rangs de l'armée ne constitue pas moins un véritable tremplin professionnel pour nombre d'individus qui cherchent à profiter des opportunités de reclassement dans la vie civile qu'assure la monarchie à ses vétérans. Quant à W. NEUGEBAUER, il met l'accent sur le rôle complémentaire que jouent les autorités centrales et locales en matière militaire au cours du XVIIIe s., infléchissant ainsi notablement le cliché de l'absolutisme prussien. Même sous le règne de Frédéric II, le système des états (Stände) apparaît en effet déterminant dans la mise en œuvre effective du potentiel militaire de la Prusse, tant sur un plan financier (par l'octroi de subsides) que logistique (en épargnant à la monarchie une lourde et coûteuse machine bureaucratique). Refusant également toute survalorisation de l'efficience des orientations impulsées par l'État central en matière militaire, M. SIKORA montre combien les réformes engagées après la défaite de 1806 ont fait l'objet de négociations, de variantes, de tâtonnements et d'improvisations bien éloignés de l'image téléologique d'un projet politique planifié et global, forgée à partir des guerres de Libération. À la lecture des débats parlementaires, la loi militaire de 1874 étudiée par H. MÜLLER apparaît également comme le résultat d'un compromis, cette fois entre le gouvernement et le Reichstag (en particulier la fraction nationalelibérale qui y siège), ce qui nuance sensiblement le cliché de l'autoritarisme brutal du chancelier de fer.

L'ouvrage propose enfin des pistes pour une étude des rapports entre société civile et armée en Prusse, en particulier pour le XIXe s. Comme le rappelle S. MÜLLER, la réduction des soldats prussiens à de simples instruments de répression et de réaction au cours des révolutions allemandes de 1848 est excessive. D'une part, il convient de prendre en compte les raisons de leur obéissance majoritaire aux ordres donnés (l'isolement géographique et social dans des provinces où ils étaient des étrangers, la crainte des sanctions, la loyauté à l'égard d'officiers qui envisagent l'armée comme la principale garante de la monarchie). D'autre part, une amorce de politisation des troupes - et plus encore des réservistes - est perceptible tant dans les provinces occidentales qu'orientales du royaume de Prusse : certes ponctuelle, limitée, éphémère et timide dans ses formes d'expression (participation à des réunions politiques, publication d'articles de presse, envoi de pétitions et de lettres de sollicitation au pouvoir central), elle n'en signale pas moins la diffusion des débats qui mobilisent 
l'opinion publique de l'époque jusque dans l'institution militaire. La question des réservistes, intermédiaires entre société civile et société militaire, est par ailleurs creusée par W. PETTER. Initialement conçue en 1813 comme un véritable lieu de formation militaire à grande échelle et un appoint potentiel aux armées régulières, la Landwehr se révèle finalement médiocrement opératoire en 1848. À l'issue des débats des années 1850 sur l'opportunité de son intégration dans les troupes de ligne, elle est reléguée à des fonctions subalternes de garnison et de logistique à l'arrière du théâtre des opérations militaires à l'initiative du général Albrecht von Roon, nommé en 1859 par le futur Guillaume Ier à la commission de réorganisation de l'armée où il impose ses idées grâce à l'appui de Manteuffel et Moltke. Ce n'est qu'à la fin de l'ère bismarckienne que se renouent très progressivement les liens entre l'armée régulière et la Landwehr, jusqu'à l'instauration - restée marginale - de troupes mixtes en 1912.

Aussi foisonnant dans les approches proposées que cohérent dans les thématiques traitées, volontiers iconoclaste, souvent original dans les périodes et les aspects qu'il met en lumière, mais malheureusement dépourvu de toute conclusion générale, ce livre constitue une utile étape pour une histoire globale de la Prusse, pensée comme une modulation particulière de la prégnance du militaire dans le champ politique, institutionnel, social et culturel que constitue l'Europe des XVIIIe et XIXe s.

Bertrand GOUJON (EA 2616 - CERHIC, Université de Reims Champagne-Ardenne) 